\title{
The use of roll vein graft in treatment of neuroma in continuity in sensory nerves
}

\author{
Khaled M Elsherbiny, MD; Eman Yahya Sadek, $M D$; \\ Ayman A Abdalsalam Elmeligy, MD
}

\author{
Department of Plastic \& Reconstructive Surgery, Ain Shams University, \\ Cairo, Egypt.
}

\begin{abstract}
A neuroma-in-continuity is a neuroma that results from failure of the regenerating nerve growth cone to reach peripheral targets and remain lost in the extraepineural environment. This is attributed to scar tissue formation which occurs at the site of nerve transection. The supporting structure of the nerve may be preserved. The nerve no longer functions properly. There may be local tenderness, disabling pain, hyperesthesia of the overlying dermatome and functional impairment. Neuroma formation is a major problem in nerve surgery and consensus about its treatment has not been reached. One of well-established techniques for treatment of such condition is resection of the neuroma and repair with interpositional nerve graft which may restore some degree of nerve function. The major drawback of this technique is that it sacrifices the remaining viable axons present in neuromas in-continuity. Roll vein graft technique was used in this study in 11 patients with neuroma-in-continuity. Exploration and neurolysis were done with $3.5 \mathrm{X}$ magnification. Vein graft was rolled around the neuroma site. Postoperative follow up has shown marked relief of symptoms as regards spontaneous pain and hyperesthesia. Results suggest the success and ease of this technique in treatment of neuroma-in-continuity.
\end{abstract}

\section{Introduction:}

A neuroma is a disorganised fibroneural tissue mass containing axons, connective tissue, and different types of cells such as Schwann's cells, macrophages, fibroblasts, and myofibroblasts which have contractile properties and are thought to contribute to neuroma pain by causing the collagen matrix to contract around unmyelinated nerve fibres. ${ }^{1,2}$ Neuromas are classified into three types; neuromas in-continuity, neuromas in completely severed nerves, and amputation neuromas. ${ }^{3}$ Neuroma in-continuity is considered as a partial nerve injury which accounts for 60 to $70 \%$ of all peripheral nerve injuries. It is usually seen in contusions, stretch or traction injuries, gunshot wounds, fractures adjacent to nerves, and even some sharp lacerations. ${ }^{3}$ Supporting structure of the nerve is more or less preserved. The actual nerve fibres are damaged and undergo degeneration. The regenerating nerve growth cone fails to reach peripheral targets due to external compression by scar formation So, the nerve no longer functions properly. ${ }^{4}$ Mackinnon has considered neuroma in continuity to belong to the sixth degree nerve injury, in which all degrees of nerve injury, from normal to neurotemesis, may coexist within a scarred nerve. 5 Neuromas-in-continuity can be subdivided into spindle neuromas with intact perineurium, and lateral neuromas that occur after partial disruption of the perineurium and after nerve repairs. ${ }^{3,6-8}$

A patient with neuroma in continuity usually complains of change of character of pain after a period of 3-6 weeks after nerve injury. The pain is usually more localized and burning in nature. There is an associated area of diminished sensation. Palpation along the course of sensory nerve may reveal subcutaneous nodule. ${ }^{9}$ Tinel's sign is useful in examination of a neuroma where gentle tapping with the end of a pen over the course of sensory nerve could produce a sensation similar to mild electric shock over the site of neuroma. 
Diagnostic nerve blocks may be helpful in cases of digital amputation neuroma. A variety of intraoperative histological staining and electrodiagnostic techniques had been suggested as a mean of identifying functioning and non-functioning fasclies. ${ }^{10}$ Hazari and Elliot suggested a classification which helps to analyze the subjective symptoms experienced by patients suffering from neuroma. Patients' complaints can be grouped broadly under four headings: 1) Spontaneous pain is pain experienced without external stimulation of the nerve. 2) Pain on pressure is pain experienced when any object touches the skin overlying the painful nerve. 3) Pain on movement is pain experienced on movement of joints or tendons adjacent, and probably tethered by scar, to the painful nerve. 4) Hypersensitivity is the experience of an unpleasant sensation on light touch of the skin overlying a neuroma. ${ }^{11}$

Various techniques were described for treatment of neuromas-in-continuity. They include chemical methods, microsurgical techniques and burying into muscle and bone. ${ }^{10}$ Microsurgery techniques for treatment of neuroma-in-continuity includes neurolysis and neuroma resection with interposition nerve grafting 11,12 and end-to side nerve grafting. ${ }^{13-16}$

Interposed vein conduit graft is used in treatment of neuroma in continuity. It could eliminate symptoms of painful neuroma of sensory nerves of the hand and prevent recurrence after excising. ${ }^{17}$ The future management of neuroma in continuity may be achieved chemically. Experimental work on suicide axonal transport immunolesioning agent aimed at inhibition of neuroma-in-continuity formation after controlled peripheral nerve damage. ${ }^{4}$

\section{Patients and methods}

This study was conducted from June 2006 to August 2007. It includes 11 patients (nine males and two females) suffering from neuroma in continuity due to previous trauma. Six patients had neuroma along the superficial radial nerve at dorsum of the lower third of the forearm and hand. Five patients had neuroma along digital nerves. All patients were suffering from spontaneous pain, pain on pressure, pain on movement, hypersensitivity and positive Tinel's sign along nerve course at the dorsum of the hand and lower third of the forearm or fingers. The most painful point and the area of hypersensitivity were marked. The previous criteria were assessed pre and postoperatively. Follow up was done one week, one month, 3 and 6 months.

\section{Technique:}

Exposure was done through the scar of previous trauma. Dissection was done guided by preoperative marking of the most painful site in the area Figure(1). The neuroma is identified as a fusiform swelling along course of the nerve Figure(2). Considerable segments of the nerve which appear to be normal and non-scarred are dissected both proximally and distally to the neuroma. Minimal internal neurolysis of neuroma was done using $3.5 \mathrm{X}$ magnification. All fibrous tissue within and around the neuroma are removed. Transection of continuous fascicles passing through the neuroma must be avoided. Nerve branches running toward the skin are ligated.

A vein graft is harvested from the same surgical field or by adding minimal extension of incision Figure(3). It should measure double the length of the neuroma. The vein is opened longitudinally Figure(4). Then it is rolled around the nerve starting from a normal point proximal to the neuroma and ends at a normal point distal to the neuroma. Intima of the vein should face the epineural surface. Vein is kept in place by using 8-0 Ethilon sutures which were taken within the outer surface of the vein Figure(5). Figure(6) is a diagram showing all the steps of the technique. Closure of the skin is done. No splint is used. 


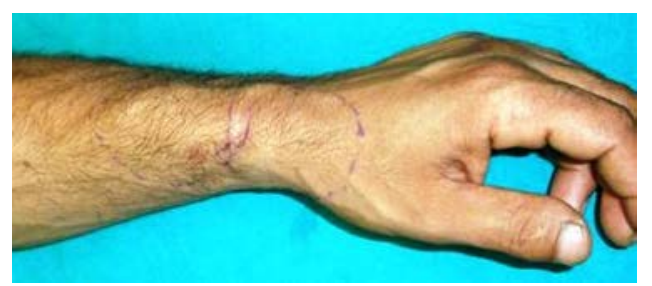

Figure (1): Scar of previous trauma with marking of the area that elicits pain.

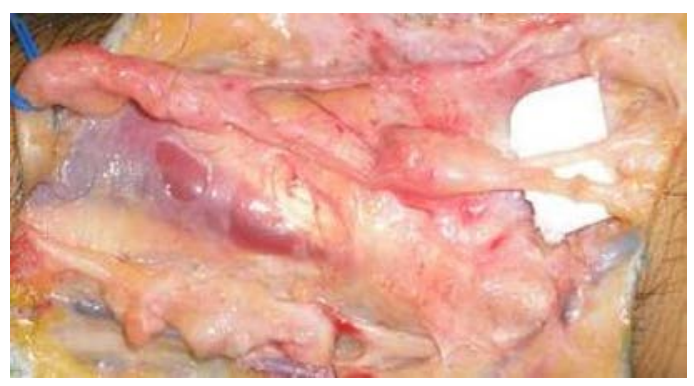

Figure (2): NIC is identified as a fusiform swelling along the superficial radial nerve.

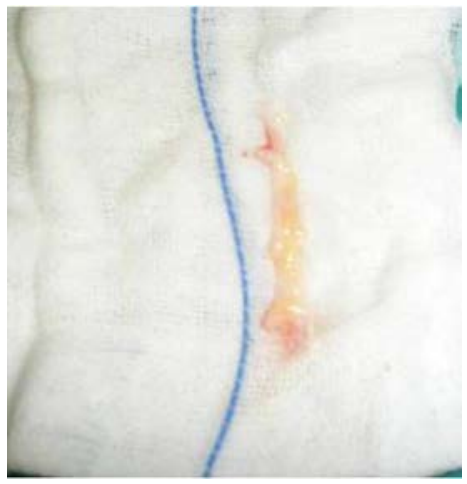

Figure (3): Vein graft is harvested.

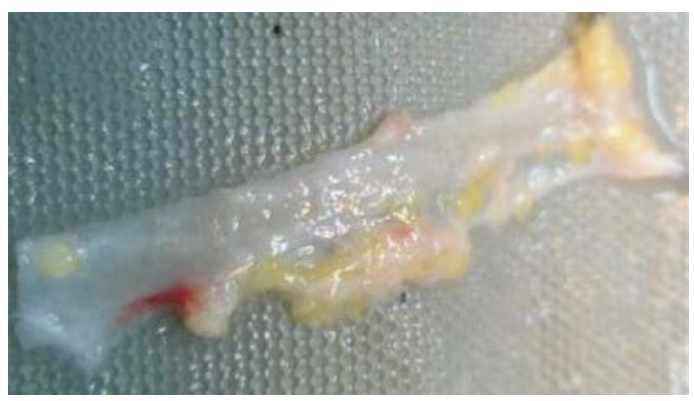

Figure (4): Longitudinal incision of the harvested vein graft.

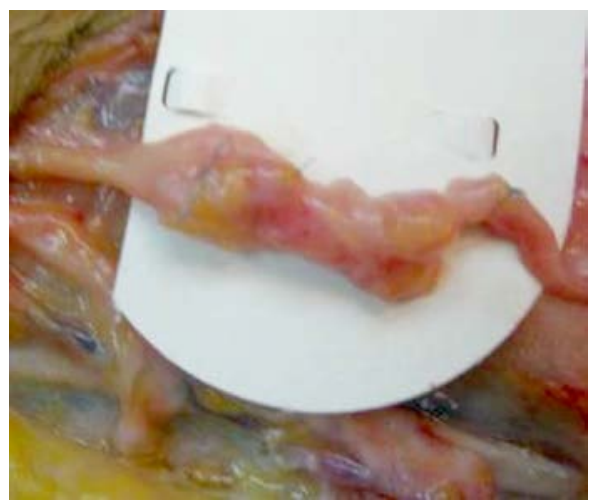

Figure (5): The incised vein graft is rolled around the neuroma and nerve distally and proximally. It has been secured by 8-0 Ethilon sutures. 


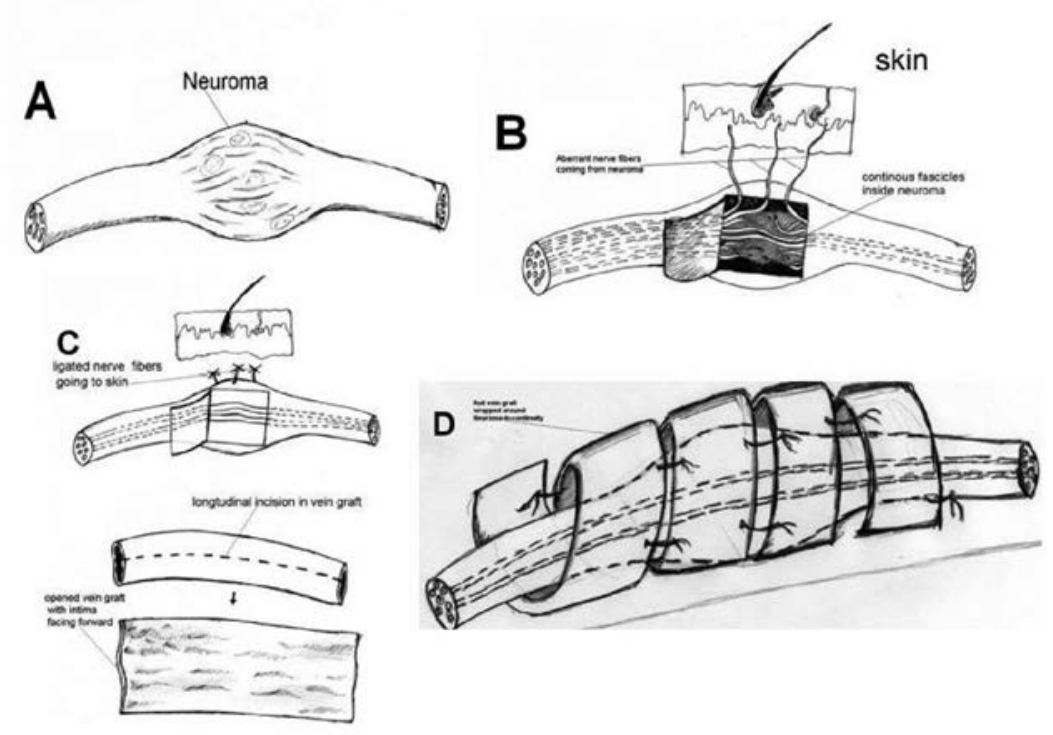

Figure (6): Steps of Roll Vein Graft Technique in treatment of neuroma in continuity. A) Neuroma along the course of a nerve, B) Internal neurolysis of neuroma reveals continuous uninterrupted branches, $C$ ) Nerve branches running toward the skin are ligated, D) The vein is splitted longitudinally and rolled around the nerve and secured by sutures.

\section{Results:}

During the first few days the patient's comments were not reliable because of postoperative edema and pain. After one week, spontaneous pain is absent, however pain on pressure, pain on movement and hypersensitivity were less. Tinel's sign was still present. At one month hypersensitivity and Tinel's sign were completely eliminated in 9 patients, the remaining 3 patients still had hypersensitivity and positive Tinel's sign. At 3 months all symptoms and signs were gone in all patients. There was no complaint of decreased or absent sensation postoperatively. Recurrence was not recorded in any of the patients.

\section{Discussion:}

Neuroma is formed in cases of non repaired transected, lacerated, avulsed or compressed nerve. Nerve endings and the sprouting axons are unable to reach the distal endoneural tubes. Pathology of NIC may be different than other types of neuroma. However prevention of neuromas can be achieved by immediate nerve repair. ${ }^{10}$ Microsurgery techniques for treatment of NIC includes neurolysis and neuroma resection with interposition nerve grafting, ${ }^{11,12}$ and end-to side nerve grafting. ${ }^{13-16}$ This type of surgery is selected when the diagnosis is clinically confirmed. This may need a reasonable period of follow-up after nerve injury. It may restore some degree of nerve function, but may also cause microvascular damage, sacrifice of remaining viable axons, formation of intraneural scars and formation of few small neuromas- in-continuity at the site of the repair with of axons being trapped at the repair site. ${ }^{18,19}$

In our technique preservation of intact axons avoid most of these drawbacks. Studies were conducted aiming to prevent and treat neuromas. Neuroma formation and neuropathic pain can be reduced by inactivation of neurotropins which are produced by the surrounding tissues. We propose that the use of rolled vein graft constitute a mechanical barrier against these neurotropins. Certain techniques aim to protect the injured or scarred nerve by its transposition into or beneath healthy tissue. Pronator quadratus muscle flap was used as a bulky coverage for treatment of neuroma in continuity at the wrist. ${ }^{9}$ Flexor digitorum superficialis was used in treatment of neuroma in continuity of the median nerve. The daily disabling pain has been significantly reduced. Still it is a local flap with few morbidity. In comparison to rolled vein graft these techniques are more difficult and time consuming. Digital nerves were covered with 
intrinsic muscle flaps after neurolysis to obtain relief of symptoms. All patients had significant relief of their symptoms. ${ }^{21}$ However, it is not expected to regain transmission along the nerve.

Ignatiadis et al had described a new technique using an epineural flap to bridge a short nerve defect after excision of NIC. They preserved the epineural sleeve and created two longitudinal epineural flaps. They reported good results. Their technique includes resection of already working axons. ${ }^{22}$

In our study we preserve these working axons which can be considered a real advantage of roll vein graft technique. Vein graft has been used as a conduit after excising the neuroma and bridging the gap. Symptoms were eliminated, and in combination with the return of adequate sensibility, ${ }^{17,23}$ it was effective in eliminating symptoms of painful neuroma of sensory nerves of the hand and preventing recurrence. The vein graft may be considered as both conduit and an insulator from the injurious inflammatory and scarring surrounding.

Other studies have been done to detect the advantages of use of vein grafts in neuroma. An experimental study was done to compare capping of the transected nerve ending with a free vein graft with non covered transected nerves. Histological and immunofluorescence analysis revealed better organization of nerve axons and better myelination in the vein capped group versus control group. ${ }^{23}$ Two postulations were introduced that agree with results obtained using rolled vein graft technique. The first is mechanical isolation from the surrounding inflammation and scar tissue which will decrease compression effect of fibrous tissues formed. These fibrous tissues were incriminated to be the cause of both pain and hypersensitivity. The second is that the vein graft decreases the effects of distal and surrounding growth factors leading to attenuated regenerative process which will help in less formation of disorganized new nervous tissue.

In cases where neuroma in continuity is associated with tendon repair the use of the present technique has an advantage over excision of neuroma and nerve repair as there is no need for splint and early physiotherapy can be done decreasing the incidence of fibrosis.

\section{Conclusion and recommendation:}

Severe disabling pain which impairs hand functions has been eliminated by using this technique. This is crucial theme in case of neuromas in hand especially in manual workers which lead to early return to work. This technique is easy to perform, easy to learn and with short operative time. It preserves intact axons leading to preservation of sensation. The vein graft is harvested from the same surgical field as subcutaneous veins are easily accessible and available. Further studies may be directed to compare the degree of return of sensation after long duration in cases of NIC treated by rolled vein graft versus resection and nerve repair.

\section{References}

1- Chen ZL, Yu WM, Strickland S: Peripheral regeneration. Ann Rev Neurosci 2007; 30: 209-233.

2- Ide C: Peripheral nerve regeneration. Neurosci Res 1996; 25(2): 101-121.

3- Kline DG: Timing for exploration of nerve lesions and evaluation of the neuroma-incontinuity. Clin Orthop 1982; 163: 42-49.

4- Mavrogenis AF, Pavlakis K, Stamatoukou A, Papagelopoulos PJ, Theoharis S, Zoubos $\mathrm{AB}$, et al: Current treatment concepts for neuromas-in-continuity. Injury 2008; 39 (3 Suppl): 43-48.

5- Mackinnon SE: Surgical management of the peripheral nerve gap. Clin Plast Surg 1989; 16: 587-603.

6- Kenzora JE: Symptomatic incisional neuromas on the dorsum of the foot. Foot Ankle 1984; 5(1): 2-15.

7- Martinelli P, Poppi M, Gaist G, Padovani R, Pozzati E: Post traumatic neuroma of the median nerve: A cause of carpal tunnel syndrome. Eur Neurol 1985; 24(1): 13-15.

8- McGrath MH, Polayes IM: Posttraumatic median neuroma: A cause of carpal tunnel syndrome. Ann Plast Surg 1979; 3(3): $227-$ 230.

9- Thomas E, Trumble H, Christopher A: Nerve repair and reconstruction. Hand Clinics 2000; 16: 67-73.

10-William BN, Richard GE: Painful neuromas of upper extremity and postneurectomy pain. In: Management of peripheral nerve problems. George E, Omer (Editors); WB 
Saunders (Publisher); 1998; p.146-151.

11-Hazari A, Elliot D: Treatment of endneuromas, neuromas-in-continuity and scarred nerves of the digits by proximal relocation. J Hand Surg 2004; 29(4): 338350.

12-Clarke HM, Al-Qattan MM, Curtis CG, Zuker RM: Obstetrical brachial plexus palsy: Results following neurolysis of conducting neuroma-in-continuity. Plast Reconstr Surg 1996; 97: 974-984.

13-Capek L, Clarke HM, Curtis CG: Neuromain-continuity resection: Early outcome in obstetrical brachial plexus palsy. Plast Reconstr Surg 1998; 102: 1555-1562.

14-Shah MH, Kasabian AK, Karp NS, et al: Axonal regeneration through an autogenous nerve bypass: An experimental study in the rat. Ann Plast Surg 1997; 38 (4): 408415.

15-Kasabian A, Karp N, Margiotta M: Treatment of a neuroma-incontinuity of the peroneal nerve with nerve bypass grafts: A case report. Ann Plast Surg 1999; 42: 449-451.

16-Aszmann OC, Korak KJ, Rab M, Grünbeck M, Lassmann H, Frey M: Neuroma prevention by end-to-side neurorrhaphy: An experimental study in rats. $J$ Hand Surg Am, 2003; 28: 1022-1028.

17-Malizos KN, Dailiana ZH, Anastasiou EA, Sarmas I, Soucacos PN: Neuromas and gaps of sensory nerves of the hand: management using vein conduits. Am J Orthop 1997; 26 (7): 481-485.
18-Low CK, Chew SH, Song IC, Ng TH, Low YP: End-to-side anastomosis of transected nerve to prevent neuroma formation. Clin Orthop 1999; 369: 327-332.

19-Gentili F, Hudson AR, Kline DG, Hunter D: Morphological and physiological alterations following internal neurolysis of normal rat sciatic nerve. In: Posttraumatic peripheral nerve regeneration: Experimental basis and clinical implications. Gorio A, Millesi H, Mingrino S (Editors); New York: Raven Press (Publisher); 1981.

20-Goth D: Animal experiment studies of neurolysis of peripheral nerves. Handchir Mikrochir Plast Chir 1987; 19: 212-216 (Abstract).

21-Gasse N, Lepage D, Rochet S, Tropet Y, Garbuio P, Obert L: The flexor digitorum superficialis flap in treatment of neuroma in continuity of the median nerve. Chir Main 2009; 24 (Abstract).

22-Ignatiadis IA, Yiannakopoulos CK, Avram AM, Gerostathopoules NE: Posttraumatic neuroma of the radial nerve treated with an autogenous epineural conduit technique. A case report. Microsurgery 2009; 29(2): 133-135.

23-Galeano M, Manasserie B, Risitano G, Geuna S, Di Scipio F, La Rosa P, Delia G, D'Alcontres FS, Colonna MR: A free vein graft cap influences neuroma formation after nerve transection. Microsurgery 2009; 29 (7): 568-572. 